\title{
Hypertrophic olivary degeneration in a 16-year-old girl after subtotal surgery of a brainstem pilocytic astrocytoma - a case report
}

\author{
Paulina Sobieraj ${ }^{1 B, D, F}$, Marek Duczkowski ${ }^{1 B, D, E, F}$, Iwona Terczyńska ${ }^{2 B}$, Agnieszka Duczkowska ${ }^{1 E, F}$, \\ Katarzyna Krupa ${ }^{1 \mathrm{~B}}$, Ewa Krupska" ${ }^{1 \mathrm{~F}}$, Monika Bekiesińska-Figatowska ${ }^{1 \mathrm{E}, \mathrm{F}}$ \\ 'Department of Diagnostic Imaging, Institute of Mother and Child, Warsaw, Poland \\ ${ }^{2}$ Clinic of Neurology of Children and Adolescents, Institute of Mother and Child, Warsaw, Poland
}

\section{Abstract}

Purpose: Hypertrophic olivary degeneration (HOD) is a unique neurological condition caused by interruption of the dentato-rubro-olivary pathway, also known as the triangle of Guillain and Mollaret. Magnetic resonance (MR) imaging is the best modality to diagnose both the degeneration of the inferior olivary nucleus and the underlying cause.

Case report: We describe a case of a unilateral HOD in a 16-year-old girl several months after a subtotal excision of a brainstem pilocytic astrocytoma. Taking into account the patient's history, tumour recurrence must have been considered, but the typical location and MR morphology, as well as the time of occurrence after brainstem surgery, contributed to the diagnosis of HOD. The causative factor was the interruption of the central tegmental tract, which forms one arm of the Guillain and Mollaret triangle. Additionally, this is an interesting case of a child, who stayed in a coma for several months following brainstem surgery, but finally was discharged home with only minor neurological defects and returned to normal life.

Conclusions: Hypertrophic olivary degeneration is an infrequent neurological condition, especially in the paediatric population. Nevertheless, it should be considered when interpreting late postoperative scans of children with a history of a brain tumour.

Key words: MRI, hypertrophic olivary degeneration, brainstem pilocytic astrocytoma.

\section{Introduction}

Hypertrophic olivary degeneration (HOD) is a unique neurological condition. It is caused by interruption of the dentato-rubro-olivary pathway, also known as the triangle of Guillain and Mollaret, formed by the inferior olivary nucleus (ION) in the medulla, the ipsilateral red nucleus $(\mathrm{RN})$ in the midbrain, and the contralateral dentate nucleus (DN) in the cerebellum. Magnetic resonance imaging (MRI) is the best modality to diagnose both the degeneration of the ION and the underlying cause. Although there are many possible causative factors, such as ischaemic infarction, haemorrhage, demyelination, tumour, trauma, or surgery, HOD is an infrequent condition and there are few reports in the paediatric population. We therefore consider our case as worth publishing.

\section{Case report}

A 16-year-old girl was referred for a brain MR scan to the Department of Diagnostic Imaging of the Institute of Mother and Child. She fell into a coma almost 13 months earlier, after a subtotal excision of a brainstem pilocytic astrocytoma. She remained without verbal contact, responded only to simple verbal commands and to pain, and showed no neurological improvement over time. Her previous brain MR studies were performed outside our institution prior to and shortly after surgery. The preop-

Correspondence address:

Marek Duczkowski, Department of Diagnostic Imaging, Institute of Mother and Child, Warsaw, Poland, e-mail: duczkom@gmail.com

Authors' contribution:

A Study design · B Data collection · C Statistical analysis · D Data interpretation - E Manuscript preparation · F Literature search · G Funds collection 
erative scan revealed a tumour with typical features of pilocytic astrocytoma, localised in the right dorsal part of the brainstem (Figure 1). The early postoperative scan (five days post-surgery) showed no clear evidence of a residual enhancing tumour (Figure 2). The subsequent MRI performed at our institution, apart from postoperative changes in the posterior fossa, revealed a region of volume increase and high signal intensity on T2-weighted (T2W) fast-spin echo (FSE) and fluid attenuation inversion recovery (FLAIR) images involving the right anterolateral aspect of the medulla at the level of ION (Figure 3). This lesion was absent on both previous studies (Figures 1 and 2). Taking into account the patient's history, the above-mentioned imaging features raised at first the suspicion of tumour recurrence on a late postoperative scan. However, the typical location and MR morphology, as well as the time of occurrence in the patient, who underwent brainstem surgery several months earlier, contributed to the diagnosis of HOD. Additionally, there was no evidence of restricted diffusion and of contrast enhancement.
Two months later, the girl came out of the coma and was discharged home in full verbal and logical contact, with no intellectual disabilities and only mild spastic quadriparesis. She returned to normal life and continued her education at school.

\section{Discussion}

Hypertrophic olivary degeneration is a unique neurological condition characterised by changes in the ION, caused by interruption of the dentato-rubro-olivary pathway, also known as the Guillain and Mollaret triangle or myoclonic triangle (palatal myoclonus is a classical clinical feature). The three vertices of this triangle, which is responsible for spinal cord motor activity modulation, are: the ION, the ipsilateral RN, and the contralateral DN (Figure 4) [1,2]. These vertices are connected by efferent fibres, forming tracts. Any interruption of the tract between the RN and the ipsilateral ION (central tegmental tract) or between the RN and the contralateral DN (superior cerebellar
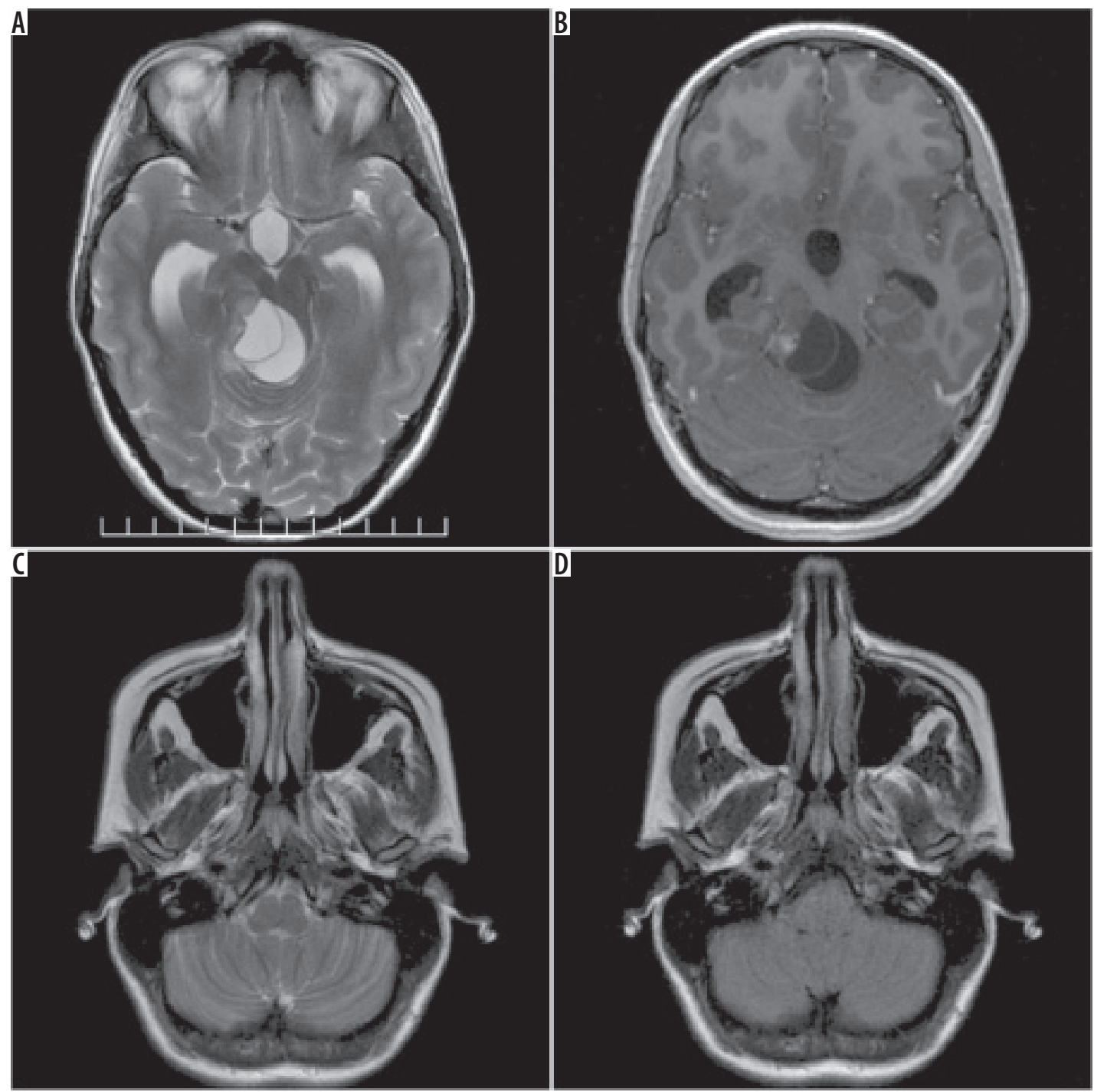

Figure 1. Preoperative magnetic resonance study. Typical features of a pilocytic astrocytoma of the right dorsal part of the brainstem: A) FSE/T2, axial plane, B) FSE/T1 post-Gd, axial plane. No changes at the level of the inferior olivary nuclei: C) FSE/T2, axial plane, D) FLAIR/T2, axial plane 

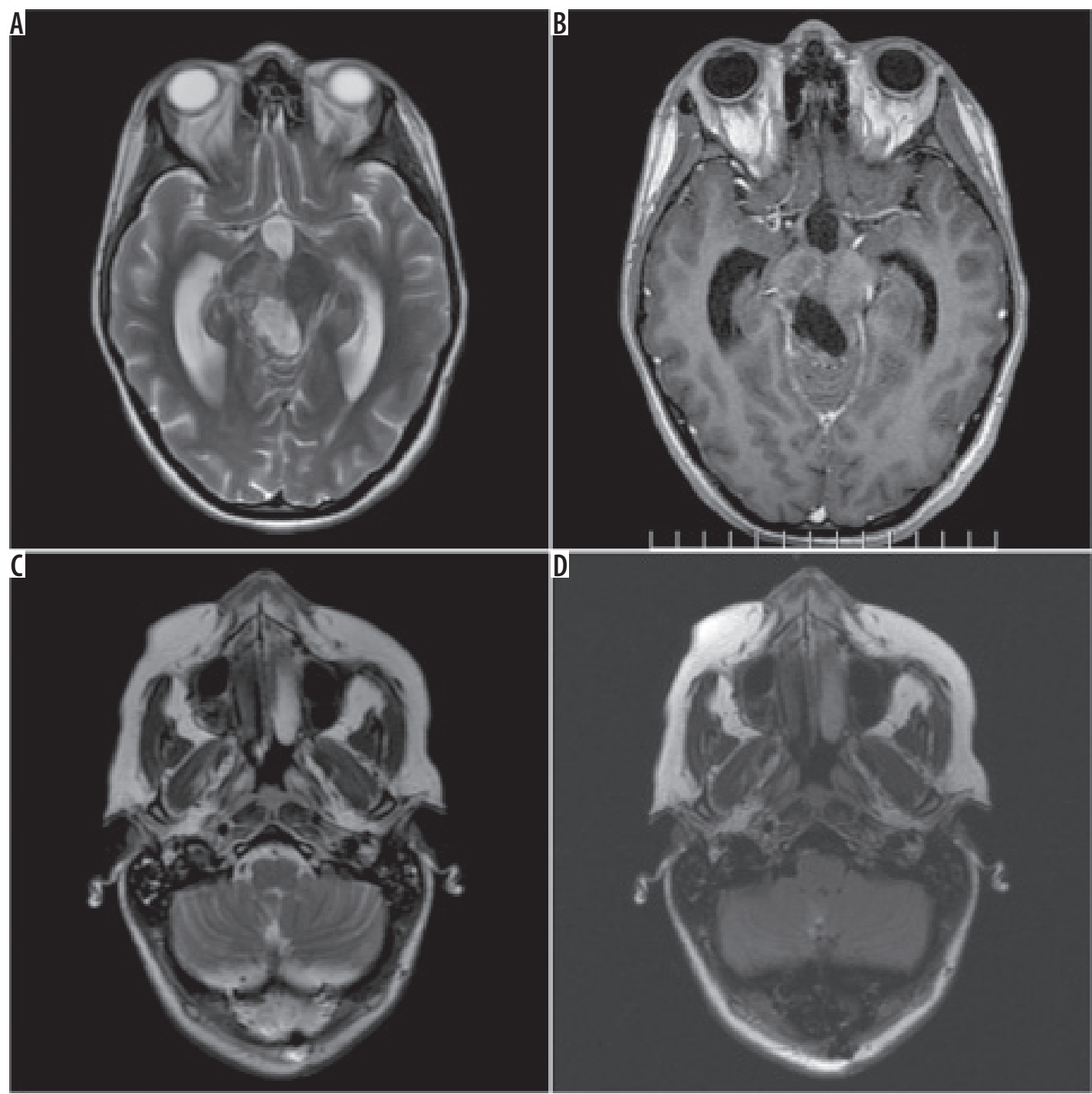

Figure 2. Magnetic resonance study 5 days after subtotal excision of a tumour. The post-resection cavity, enlarged lateral ventricles: A) FSE/T2, axial plane, B) FSE/T1 post-Gd, axial plane. No changes at the level of the inferior olivary nuclei: C) FSE/T2, axial plane, D) FLAIR/T2, axial plane

peduncle), leads to the degeneration of the ION. Importantly, an isolated insult to the tract connecting the ION and the contralateral DN (inferior cerebellar peduncle) does not cause HOD. This is due to the indirect connection between these nuclei [3].

Hypertrophic olivary degeneration is an infrequent condition, especially in the paediatric population. So far there were only 14 publications describing a total of 39 cases of HOD in children after posterior fossa surgery [4].

Interruption of the Guillain and Mollaret triangle may occur as a result of either focal lesions, such as ischaemic infarction, haemorrhage, demyelination, tumour, trauma, or surgery [3].

There are three patterns of HOD, depending on the location of the primary lesion: 1) ipsilateral to the primary lesion, 2) contralateral to the primary lesion, and 3) bilateral (Figure 5) [5,6].

Degeneration of the ION, resulting initially in hypertrophy rather than atrophy, makes it a unique type of trans-synaptic degeneration [7]. Histologically, the olivary enlargement is due to vacuolar cytoplasmic degeneration and increased number of astrocytes. Goto et al. distinguished six stages of HOD: 1) no olivary changes, 2) olivary amiculum degeneration, 3) olivary hypertrophy, 4) culminant olivary enlargement, 5) olivary pseudohypertrophy, and 6) olivary atrophy [8].

The imaging features are often seen several months after the insult and persist indefinitely. MRI is the best imaging tool. Typically, the ION enlarges and becomes hyperintense on T2W images. Imaging findings depend on the stage of degeneration. On MRI three stages can be distinguished: 1) hyperintensity without hypertrophy (within the first six months after insult), 2) hyperintensity with hypertrophy (between six months and 3-4 years), and 3) hyperintensity without hypertrophy again (can persist indefinitely) [2,9]. Similar features can also be appreciated on T2-FLAIR images and on proton-density (PD) images [10].

The differential diagnosis of T2-hyperintensity in the anterior part of the medulla also includes: 1) demyelina- 

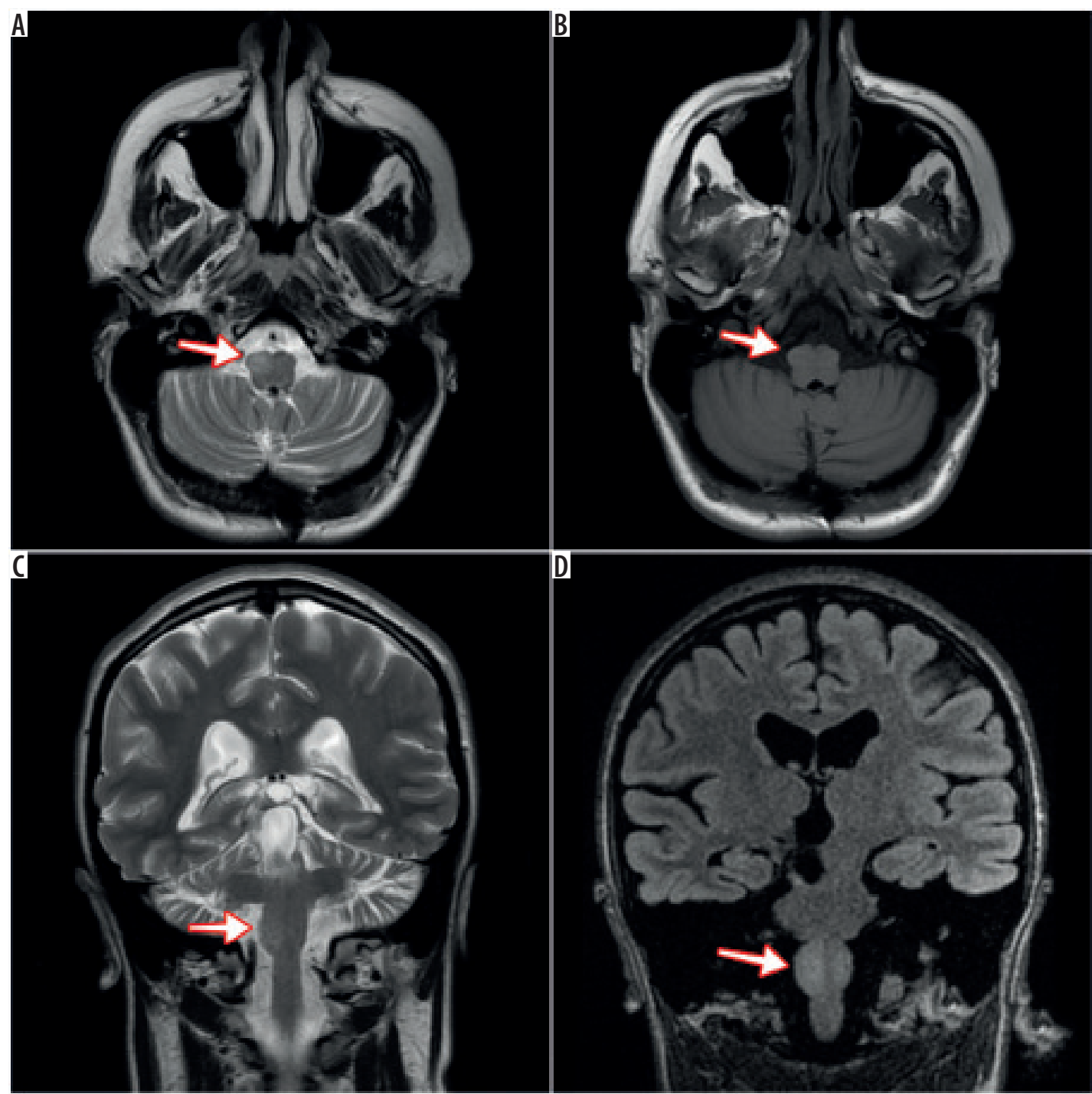

Figure 3. Magnetic resonance study almost 13 months after surgery. Volume and signal increase at the level of the right ION - features of unilateral hypertrophic olivary degeneration (arrows): A) FSE/T2, axial plane, B) FLAIR/T2, axial plane, C) FSE/T2, coronal plane, D) 3D/Cube FLAIR, coronal plane

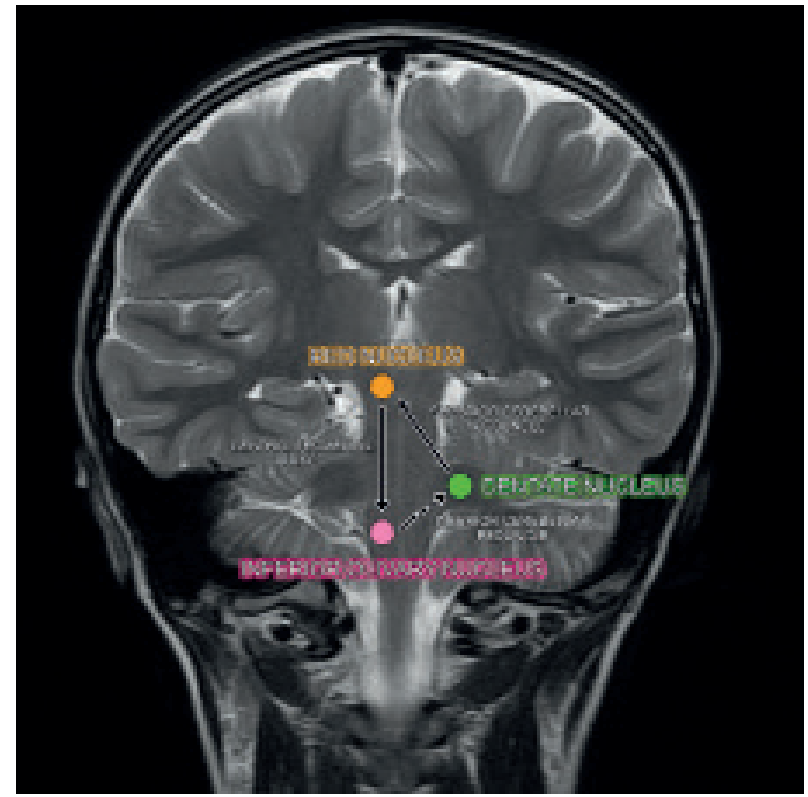

Figure 4. Schematic diagram of Guillain-Mollaret triangle on a T2-weighted coronal image

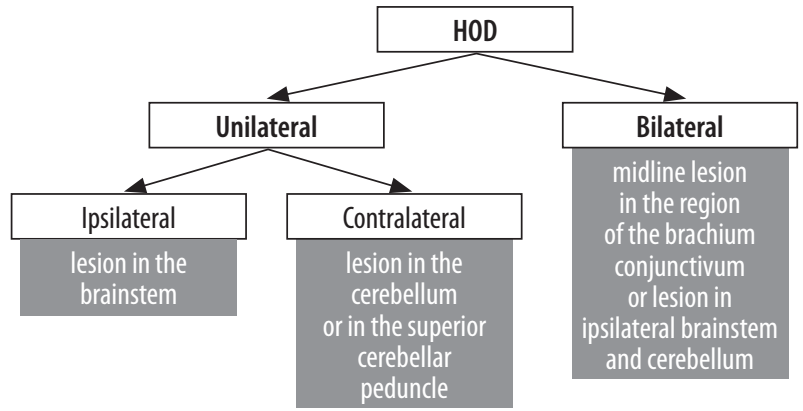

Figure 5. Patterns of hypertrophic olivary degeneration (HOD)

tion related to multiple sclerosis, 2) tumours, such as astrocytoma, lymphoma, or metastasis, 3) lesions involving the corticospinal tracts (Wallerian degeneration, adrenoleukodystrophy, amyotrophic lateral sclerosis), 4) vertebrobasilar perforating artery infarct, and 5) infectious/ inflammatory processes, such as tuberculosis, sarcoidosis, and HIV infection [11].

In the case of our patient, the unilateral (right-sided) enlarged and hyperintense ION was first observed on 
MRI several months after a subtotal surgery of pilocytic astrocytoma of the right dorsal part of the brainstem. The prior MR examinations performed before and shortly after surgery showed no changes in the location of ION. Taking into account the patient's history, the above-mentioned imaging features raised at first the suspicion of tumour recurrence. However, the typical location and MR morphology as well as the time of occurrence in the patient, who underwent brainstem surgery several months earlier, contributed to the diagnosis of HOD. Surgical interruption of the right central tegmental tract (connecting the right $\mathrm{RN}$ with the ipsilateral ION) resulted in the ipsilateral HOD.

\section{Conclusions}

Hypertrophic olivary degeneration is an infrequent neurological condition, especially in the paediatric population. Nevertheless, it should be considered when interpreting late postoperative scans of children with a history of a brain tumour.

\section{Conflict of interest}

The authors report no conflict of interest.

\section{References}

1. Harter DH, Davis A. Hypertrophic olivary degeneration after resection of pontine cavernoma. Case illustration. J Neurosurg 2004; 100: 717 .

2. Sanverdi SE, Oguz KK, Haliloglu G. Hypertrophic olivary degeneration in children: four new cases and a review of the literature with an emphasis on the MRI findings. Br J Radiol 2012; 85: 511-516.

3. Van Eetvelde R, Lemmerling M, Backaert T, et al. Imaging features of hypertrophic olivary degeneration. J Belg Soc Radiol 2016; 100: 71.

4. Ballestero MFM, Viana DC, Teixeira TL, et al. Hypertrophic olivary degeneration in children after posterior fossa surgery. An underdiagnosed condition. Childs Nerv Syst 2018; 34: 409-415.

5. Sarawagi R, Murugesan A. Hypertrophic olivary degeneration a report of two cases. J Clin Imaging Sci 2015; 5: 8.

6. Sen D, Gulati YS, Malik V, et al. MRI and MR tractography in bilateral hypertrophic olivary degeneration. Indian J Radiol Imaging 2014; 24: 401-405.

7. Kitajima M, Korogi Y, Shimomura O, et al. Hypertrophic olivary degeneration: MR imaging and pathologic findings. Radiology 1994; 192: 539-543.

8. Goto N, Kaneko M. Olivary enlargement: chronological and morphometric analyses. Acta Neuropathol 1981; 54: 275-282.

9. Goyal M, Versnick E, Tuite P, et al. Hypertrophic olivary degeneration: metaanalysis of the temporal evolution of MR findings. AJNR Am J Neuroradiol 2000; 21: 1073-1077.

10. Blanco Ulla M, López Carballeira A, Pumar Cebreiro JM. Magnetic resonance imaging of hypertrophic olivary degeneration. Radiologia 2015; 57: 505-511.

11. Krings T, Foltys H, Meister IG, et al. Hypertrophic olivary degeneration following pontine haemorrhage: hypertensive crisis or cavernous haemangioma bleeding? J Neurol Neurosurg Psychiatry 2003; 74: 797-799 